\title{
EI Mapa Conceptual como Estrategia Pedagógica en el Aprendizaje de Conceptos Disciplinares de Economía
}

\author{
The Conceptual Map as a Pedagogical Strategy in the Learning of Disciplinary \\ Concepts of Economics
}

German Enrique Cárdenas-Contreras ${ }^{1}$

\section{a EDICIÓN: \#-CIVTAC}

Recibido: 27/enero/2021

Aceptado: 8/abril/2021

Publicado: 16/abril/2021

País

${ }^{1}$ Colombia

\section{IIII Institución}

${ }^{1}$ Universidad de Pamplona

\section{$\chi_{\varnothing}$ Correo Eletrónico}

'german.cardenas@unipamplona.e du.co

\section{iD ORCID}

${ }^{1}$ https://orcid.org/0000-0002-1343-935X

\section{Citar así: HAPA / IEEE}

Cárdenas-Contreras, G. (2021). El mapa conceptual como estrategia pedagógica en el aprendizaje de conceptos disciplinares de Economía. Revista TecnológicaEducativa Docentes 2.0, 11(1), 74-79. https://doi.org/10.37843/rted.v11i1.194

G. Cárdenas-Contreras, "El mapa conceptual como estrategia pedagógica en el aprendizaje de conceptos disciplinares de Economía", RTED, vol. 11, n. ${ }^{\circ}$ 1, pp. 7479, abr. 2021.

\section{Resumen}

En la actualidad, existe gran preocupación mundial por la necesidad de transformar los modelos educativos. Por ello, la presente investigación se orientó al análisis de la incidencia de la teoría de Novak en el aprendizaje de conceptos disciplinares de la asignatura Historia Económica de Colombia en estudiantes de $4^{\circ}$ semestre del programa de Economía de la Universidad de Pamplona, Colombia. El estudio se realizó bajo un enfoque mixto, en donde la consecución del objetivo establecido se procedió a realizar un proceso de identificación de acuerdo con el aprendizaje de conceptos disciplinares en los estudiantes objeto de estudio. Así mismo, como instrumentos de recolección de información se aplicó una prueba diagnóstica por competencias, una prueba de aptitud y una entrevista final, resultados con los cuales se pudo establecer el impacto e incidencia de los mapas conceptuales en la enseñanza de conceptos disciplinares de Economía. Los resultados obtenidos dieron muestra de la importancia de la incorporación de herramientas como los mapas conceptuales para la enseñanza de contenidos teóricos, en donde se hace necesario que los docentes adapten sus métodos pedagógicos, a las necesidades de aprendizaje de sus estudiantes, buscando no solo la enseñanza de un contenido de forma mecánica, sino que más bien generando en ellos, la suficiente motivación intrínseca que les permita adquirir competencias para que puedan construir aprendizajes significativos de calidad, acorde a las exigencias en materia Económica que se presentan en el mundo actual.

Palabras clave: Mapas conceptuales, estrategia pedagógica, conceptos disciplinares, economía.

\section{Abstract}

The academic article described below is the underlying work of an investigative process aimed at analyzing the incidence of Novak's theory in the learning of disciplinary concepts of the subject Economic History of Colombia in students of the 4th semester of the Economics program of the University of Pamplona, ??Colombia, the study is of mixed approach, where in order to achieve the established objective, an identification process was carried out in accordance with the learning of disciplinary concepts in the students under study. Likewise, as instruments for collecting information, a diagnostic test by competencies, an aptitude test and a final interview were applied, results with which it was possible to establish the impact and incidence of concept maps in the teaching of disciplinary concepts of Economics. The results obtained show the importance of incorporating tools such as concept maps for teaching theoretical content, where it is necessary for teachers to adapt their pedagogical methods to the learning needs of their students, seeking not only the teaching content in a mechanical way, but rather generating in them enough intrinsic motivation that allows them to acquire skills so that they can build meaningful quality learning, according to the economic demands that are presented in today's world.

Keywords: Didactic proposal; leveling course; renewable energy; learning strategies. 


\section{Introducción}

En la actualidad, existe gran preocupación mundial por la necesidad de transformar los modelos educativos, ya que, según la Organización de las Naciones Unidas para la Educación, la Ciencia y la Cultura, Unesco (2015) "a lo largo de las dos últimas décadas ha ido surgiendo un movimiento mundial que aboga por un nuevo modelo de aprendizaje para el siglo XXI" (p. 34). Este modelo se basa en tres pilares: justificación de un nuevo modelo de aprendizaje, las competencias específicas que se necesitan para poder ser eficiente en este nuevo siglo, y la pedagogía para estimular dichos conceptos, capacidades y aprendizaje. En este orden de ideas, el presente artículo es el resultado de un proyecto investigativo realizado con el propósito de analizar la incidencia de la teoría de Novak en el aprendizaje de conceptos disciplinares en estudiantes del programa de Economía de la Universidad de Pamplona, utilizando los mapas conceptuales como herramienta de apoyo didáctico y pedagógico.

Los mapas conceptuales basados en la teoría del aprendizaje de Ausubel (1986) y desarrollada por Novak (1988) son una herramienta utilizada con bastante frecuencia para la explicación de conceptos disciplinares de diferentes áreas de estudio. Por lo tanto, se optó por seleccionar la teoría de Novak porque es necesario que el estudiante no memorice conceptos, ni resuelva problemas abstractos mediante la aplicación de métodos e información que no comprende, se requiere que adquiera y desarrolle habilidades conforme con las problemáticas y exigencias socioeconómicas y culturales que se presentan en la actualidad.

Desde la postura de Ausubel (1988) "la esencia del proceso del aprendizaje significativo radica en que nuevas ideas expresadas de una manera simbólica se puedan relacionar con aquello que ya sabe el estudiante" (p. 45) en donde se requiere que este pueda integrar los nuevos conocimientos que adquiere con los ya existentes en sus esquemas cognitivos y de pensamiento, logrando construir patrones de pensamiento de mayor complejidad.
En este orden de ideas, retomando los principios de Novak (1988) hay que mencionar que los mapas conceptuales son una herramienta de gran utilidad en los procesos de enseñanzaaprendizaje, la cual, si es utilizada de forma apropiada, permite la construcción de conocimiento, dinamizando la formación de estructuras intrapsíquicas capaces de relacionar nuevos conocimientos con los ya establecidos previamente, los cuales se verán reflejados en la calidad de las respuestas de los estudiantes durante el trascurso de su desarrollo formativo, educativo y psicosocial.

Finalmente, cabe mencionar que el artículo describe la metodología implementada para la consecución de los objetivos planteados, así mismo, y a modo de complemento se presenta la descripción de los hallazgos obtenidos en conjunción con los aspectos más relevantes posterior a la realización de las actividades orientadas al análisis de la incidencia de la teoría de Novak en el aprendizaje de conceptos disciplinares en estudiantes de cuarto semestre del programa de Economía de la Universidad de Pamplona.

\section{Metodología}

En el marco de la estructura metodológica el estudio estuvo precedido por el enfoque de investigación mixta, el cual según lo argumenta Hernández, Fernandez \& Batista (2014) "representa un conjunto de procesos sistemáticos, empíricos de investigación que implica la recolección de datos cuantitativos y cualitativos, así como su integración y discusión conjunta" (p. 128) para realizar inferencias producto de toda la información recabada (metainferencias) hecho que permite lograr una mayor comprensión del problema de estudio. Por lo tanto, la dificultad de concertar la teoría pedagógica con la realidad social de los estudiantes, la investigación educativa y la investigación-acción pedagógica pueden inspirar al docente a dominar las tensiones en el aula. La investigación-acción ofrece una salida que comienza con la crítica a la propia práctica, a través de un proceso de reflexión profundiza acerca del quehacer pedagógico. 
En relación directa con este planteamiento, Restrepo (2004) aduce que, "esta fase de deconstrucción es un proceso que trasciende la misma crítica, que va más allá de un autoexamen de la práctica, para entrar en diálogos más amplios, con componentes que explican la razón de ser de las tensiones que la práctica enfrenta" (p. 50). El diseño seleccionado estuvo fundamentó desde los principios del método hermenéutico por lo que se realizó una interpretación profunda la cual permitió sistematizar toda la información obtenida que se constituye como el producto pedagógico y académico en este esfuerzo intelectual.

La Hermenéutica es la comprensión de los fenómenos humanos y sociales, Sabino (2008) señala que "consiste en la recolección de datos directamente de los sujetos investigados o de la realidad donde ocurren los hechos (datos primarios), sin manipular ni controlar dato alguno, es decir, el investigador obtiene la investigación, pero no altera las condiciones existentes" (p.31). El diseño y procedimiento utilizado en el desarrollo de las actividades consistió en la realización de una propuesta pedagógica utilizando los mapas conceptuales como herramienta de apoyo pedagógico. La propuesta describe los propósitos, recursos, duración y objetivos pedagógicos establecidos durante el desarrollo de la actividad fundamentada en los lineamientos conceptuales y teóricos de los 3 primeros capítulos del Libro, Breve Historia Económica de Colombia.

Por todo lo dicho anteriormente, la implementación de mapas conceptuales como método de aprendizaje, resulta ser una herramienta que, además de sintetizar la información más relevante, promueve la creatividad en los estudiantes, demostrando el valor útil del aprendizaje significativo, siendo instrumentos que llevan a profundas modificaciones en la manera de enseñar, de aprender. Si se utilizan con toda su potencialidad, implica atribuir nuevos significados a los conceptos de enseñanza, aprendizaje y evaluación.

Cabe destacar que los sujetos en estudio estuvieron conformados el grupo A de Historia Económica de Colombia inscritos para el segundo semestre de 2020. La muestra de estudio se seleccionó de manera intencional a determinados sujetos siguiendo algunos criterios específicos, a este respecto Mendieta (2015) expone que, el proceso de selección de informantes no se interrumpe, sino que continúa a lo largo de toda la investigación, valiéndose de estrategias diferentes según el tipo de información que se necesita en cada momento. Como consecuencia, este tipo de procedimiento también puede definirse como fásico, dado que su desarrollo tiene lugar en más de una etapa o momento de investigación.

El procedimiento de recolección de información se realizó mediante la aplicación de una prueba diagnóstica de conocimientos, que estuvo orientada a determinar el nivel de las competencias y la calidad del conocimiento y aprendizaje de conceptos disciplinares de economía. Así mismo, se aplicó una prueba de aptitud y una prueba final tipo entrevista de validación con la cual se puedo establecer el impacto de las actividades desarrolladas y su incidencia en el aprendizaje de conceptos disciplinares en la asignatura historia económica de Colombia.

La recolección de datos es un paso obligatorio en cualquier tipo de investigación, pero en la investigación cualitativa no tiene como propósito medir las variables, sino que busca obtener información de sujetos, comunidades, contextos, cualidades, situaciones en profundidad por lo que las principales técnicas utilizadas en la investigación fueron la entrevista, la observación y el análisis documental, instrumentos que permitieron recolectar la información necesaria para el diseño y puesta en práctica de las actividades contenidas en la propuesta pedagógica.

\section{Resultados}

Los resultados indican que entre las principales falencias encontradas en los estudiantes se tiene que todos los integrantes de la muestra de estudio presentan limitaciones al momento de utilizar palabras-enlace, las cuales son las que permiten darle fluidez a la información presentada, de igual forma, presentaron conceptos muy extensos, evidenciando la falta de capacidad de síntesis en los estudiantes, así mismo, no hicieron uso de las preposiciones, las cuales son nexos que permiten 
relacionar los diferentes elementos de una oración, obteniendo oraciones faltas de sentido y coherencia, así mismo, no realizaron una correcta utilización de las líneas y flechas de enlace, obteniendo como resultado final un mapa conceptual confuso, que no cumplía con los estándares básicos de elaboración.

Los mapas conceptuales son usados para representar gráficamente el conocimiento implícito, como un concepto o teoría. De acuerdo con lo propuesto por Novak la finalidad de los mapas conceptuales se basa en representar de forma gráfica relaciones significativas entre conceptos, teniendo en cuenta que la información debe estar organizada, representada en niveles de abstracción, ubicando los conceptos generales en la parte superior y los más específicos en la parte inferior.

Así mismo, la utilización de mapas conceptuales en la actualidad, resulta ser una metodología aplicable a casi la totalidad de las ramas del saber, siendo una herramienta indispensable para representar el conocimiento adquirido, logrando obtener un verdadero aprendizaje significativo con la ejecución correcta. Es así que la implementación de mapas conceptuales como método de aprendizaje, resulta ser una herramienta que, además de sintetizar la información más relevante, promueve la creatividad en los estudiantes, demostrando el valor útil del aprendizaje significativo, así mismo, permite una interpretación constructivista de la enseñanza, la cual se une al principio de la actividad mental del estudiante, en contraposición con las prácticas cotidianas que son evidenciadas en los estudiantes del programa de Economía, en las cuales, se da prioridad al aprendizaje memorístico, obteniendo como resultado un bajo rendimiento en los procesos evaluativos de las asignaturas relacionadas.

Consistentemente con lo ya planteado Guerra (2018) argumenta que "el mapa conceptual desarrollado por Ausubel y Novak, es un dispositivo de instrucción que utiliza este aspecto de la teoría para permitir la instrucción de los alumnos; es una forma de representar las relaciones entre las ideas, imágenes o palabras" (p. 34). Por lo tanto, aprender significa adquirir información, retenerla, recuperarla en algún momento, esta perspectiva teórica considera que ciertos tipos de aprendizaje son mejor que otros; el aprendizaje por recepción resulta ser más efectivo, que el aprendizaje por descubrimiento o el aprendizaje memorístico, ver Tabla 1.

\section{Tabla 1}

\begin{tabular}{l} 
Análisis de los datos \\
\hline $\begin{array}{l}\text { Unidad Textual de Código } \\
\text { análisis }\end{array}$
\end{tabular}
me permite conocer la historia sino relacionarla con hechos actuales $y$ entender futuro hechos económicos que de una u otra manera intervienen en el desarrollo de nuestro país.

$\begin{aligned} & \text { Permite recordad de } \\ & \text { forma visual } 1\end{aligned}$
$\begin{aligned} & \text { la } \\ & \text { organización }\end{aligned}$
$\begin{aligned} & \text { y } \\ & \text { distribución de los }\end{aligned}$
conceptos, estableciendo
las claves sobre el tema
que se está aprendiendo;
de igual forma, establece
que existen entre los
mismos.

\begin{tabular}{l}
\hline Informante 2 \\
\hline $\begin{array}{l}\text { Aprender de manera A1 } \\
\text { muy rápida, resumida e }\end{array}$ \\
interesante temas que \\
han intervenido en la \\
economía de nuestro \\
país. \\
$\begin{array}{l}\text { Es un elementicativo } \\
\text { favorable muy A1 }\end{array}$ \\
instrumento Aprendizaje \\
construcción personal \\
frente a los referentes \\
conceptuales \\
notables del programa \\
académico. \\
Nota. La implementación de mapas conceptuales como \\
método de aprendizaje, resulta ser una herramienta que, \\
además de sintetizar la información más relevante, \\
elaboración propia (2020).
\end{tabular}

\section{Conclusiones}

En primera medida fue necesario identificar la organización de los conceptos disciplinares en los estudiantes en donde los resultados obtenidos con la implementación de la prueba diagnóstica por competencias 
evidenciaron que del $100 \%$ de los estudiantes, el $50 \%$ obtuvo una nota igual o superior a 4.5 , mientras que el otro $50 \%$ obtuvo una nota inferior a 4.5 , esto evidencia que no hubo ningún estudiante que pudiera resolver en su totalidad la prueba por competencias.

Por su parte, los resultados de la prueba de aptitud develó que del 100\% de los estudiantes el $87 \%$ evidenció tener una actitud emocional asertiva ante las diversas situaciones y conflictos que se pueden presentar en la vida cotidiana de todos y cada uno de ellos, siendo claro que, les afecta y preocupa el problema que se tiene en el país con respecto al conflicto y a la violencia, de igual forma, siempre intentan ser comprensivos y entender el punto de vista de las otras personas, que no siempre tienen su misma forma de pensar, así mismo, siempre se esfuerzan por hacer su mejor esfuerzo en todas las actividades que realizan día a día, dejando manifiesto de su empatía hacía las otras personas dentro de un contexto socio- temporal.

Se puede concluir que es necesario replantear las didácticas y pedagogías de enseñanza, en donde en este estudio con el uso de los mapas conceptuales de Novak como estrategia Didáctica y pedagógica se generó en lo estudiantes un aprendizaje significativo, el cual les permitió hacer una mejor selección de las ideas principales y conocimiento estableciendo relaciones coherentes y lógicas entre sí.

A pesar de todas las ventajas que representa la implementación de una metodología basada en la elaboración de mapas conceptuales como técnica de estudio, se pudo detectar en los estudiantes diversas falencias que van desde el no entendimiento de la estructura y jerarquización básica, hasta la falta de capacidad de síntesis en la relación de conceptos. Los mapas conceptuales pueden seleccionar las ideas más importantes de un determinado, o de varios temas, suministrando un resumen esquemático del mismo, por ende haciendo mucho más fácil su aprendizaje.

\section{Reconocimiento}

Agradecimientos del autor al Dr. German Amaya Franky, director de la Maestría en Educación modalidad virtual de la Universidad de Pamplona. Al Dr. José Antonio Cegarra
Guerrero, mi asesor de tesis, gracias por brindarme parte de sus conocimientos. A los compañeros maestrantes Teresa Baldion $\mathrm{y}$ Rolando Quintero por el apoyo brindado durante el proceso.

\section{Referencias}

Ausubel, D. (1988). Teoría del aprendizaje significativo. Psicología $y$ Mente. https://psicologiaymente.com/desarrollo/aprendizajesignificativo-david-ausubel

Estrada, J., \& Correa, J. (2019). El proceso enseñanzaaprendizaje y los mapas conceptuales: una reflexión desde la educación en ciencias de la salud. https://revistas.unal.edu.co/index.php/actaodontocol/articl e/view/75747

Guerra, M. (2018). La teoría del aprendizaje significativo de Ausubel. Psicoactiva. https://www.psicoactiva.com/blog/aprendizajesignificativo-ausubel/

Hernández-Sampierí, R., Fernández, C., \& Batista, P. (2014). Metodología de la Investigación Sexta Edición. Universidad de Cartagena. $\mathrm{http}: / /$ observatorio.epacartagena.gov.co/wpcontent/uploads/2017/08/metodologia-de-lainvestigacion-sexta-edicion.compressed.pdf

Mendieta, G. (2015). Informantes y muestreo en investigación cualitativa. Investigaciones Andina. https://www.redalyc.org/pdf/2390/239035878001.pdf

Novak, J. (1988). Teoría del aprendizaje significativo. [Video]. Monografias.Com.

https://www.youtube.com/watch?v=9ag2RyDdBWU\&ab _channel=FearlessRecords

Restrepo, B. (2004). La investigación-acción educativa y la construcción de saber pedagógico. Educación y Educadores.

https://www.redalyc.org/pdf/834/83400706.pdf

Sabino, C. (2008). El proceso de investigación. Ufm. Es. http://paginas.ufm.edu/sabino/ingles/book/proceso_invest igacion.pdf

Kalmanovitz, S. (2017). Breve Historia Económica de Colombia. http://kimera.com/data/redlocal/ver demos/RLBVF/VER SION/RECURSOS/REFERENCIA $\%$ 20ESCOLAR/2\%20 BIBLIOTECA\%20BASICA\%20COLOMBIANA/breve $\% 20$ historia $\% 20$ economica $\% 20 \mathrm{de} \% 20$ colombia $\% 20$ bbcc _libro_pdf_090\%20.pdf

Toro, C., Cepeda, C., \& Gil, L. (2018). Uso del mapa conceptual como estrategia didáctica para mejorar la comprensión lectora.

https://revistas.uptc.edu.co/index.php/educacion_y_cienci a/article/view/9391 
Tusa, E. (2017). Aprendizaje memorístico-significativo. https://fido.palermo.edu/servicios_dyc/publicacionesdc/vi sta/detalle_articulo.php?id_libro $=684 \&$ id_articulo $=14320$

Unesco (2015). La Educación, la Ciencia y la Cultura. https://es.unesco.org

Velasquez, L., Revilla, J., \& Guerra, M. (2018). Confección de mapas conceptuales para la enseñanza de la Química Orgánica.

http://scielo.sld.cu/scielo.php?script=sci_abstract\&pid=S2 224-54212018000300012\&lng=es\&nrm=iso

Viera, T. (2003). El aprendizaje verbal significativo de Ausubel. Algunas consideraciones desde el enfoque histórico. https://www.redalyc.org/pdf/373/37302605.pdf

Vilboa, P., Paris, M., \& Vargas, R. (2013). Aplicando mapas conceptuales como estrategia para generar un espacio de aprendizaje colaborativo http://sedici.unlp.edu.ar/bitstream/handle/10915/27545/D ocumento_completo.pdf? sequence $=1$ \&isAllowed $=\mathrm{y}$

Yaber, I., Ariza, D., \& Muñiz, J. (2008). Los mapas conceptuales como estrategia didáctica para el aprendizaje de conceptos de biología celular en estudiantes universitarios. http://manglar.uninorte.edu.co/bitstream/handle/10584/69 2/3776456.pdf? sequence $=1$ \&isAllowed $=y$

Yánez, J. (2020). A Propósito del aprendizaje: Un paralelo entre Ausubel y Feuerstein. https://pensar-lopensado.com/aproposito-del-aprendizaje-un-paralelo-entre-ausubel-yfeuerstein/ 